\title{
A Game-Theoretic Approach for Modeling Competitive Diffusion over Social Networks
}

\author{
Shahla Jafari and Hamidreza Navidi * (iD \\ Department of Applied Mathematics, Shahed University, Tehran, Iran; shahlajafari1366@gmail.com \\ * Correspondence: navidi@shahed.ac.ir
}

Received: 26 October 2017; Accepted: 23 January 2018; Published: 13 February 2018

\begin{abstract}
In this paper, we consider a novel game theory model for the competitive influence maximization problem. We model this problem as a simultaneous non-cooperative game with complete information and rational players, where there are at least two players who are supposed to be out of the network and are trying to institutionalize their options in the social network; that is, the objective of players is to maximize the spread of a desired opinion rather than the number of infected nodes. In the proposed model, we extend both the Linear Threshold model and the Independent Cascade model. We study an influence maximization model in which users' heterogeneity, information content, and network structure are considered. Contrary to previous studies, in the proposed game, players find not only the most influential initial nodes but also the best information content. The proposed novel game was implemented on a real data set where individuals have different tendencies toward the players' options that change over time because of gaining influence from their neighbors and the information content they receive. This means that information content, the topology of the graph, and the individual's initial tendency significantly affect the diffusion process. The proposed game is solved and the Nash equilibrium is determined for a real data set. Lastly, the numerical results obtained from the proposed model were compared with some well-known models previously reported in the literature.
\end{abstract}

Keywords: social network; game theory; information diffusion; Nash equilibrium; influence maximization problem

\section{Introduction}

In recent years, there has been a growing interest in studying social networks as they have widespread application in different fields such as sociology, economics, computer science, biology, and mathematics [1-6]. In every society, information is disseminated among the population by the relationships between individuals. Thus, an important part of research on social networks targets the problem of diffusion and spread of products, information, and so forth in social networks. Two early studies in this context deal with two most popular influence models called the Linear Threshold model and the Independent Cascade model by Domingos and Richardson [7] and Kempe et al. [8]. It is thought that diffusion of messages is usually more effective and convincing if messages are received from a friend rather than from a social change agent (e.g., companies) [9]. The social change agents work to support people and organizations with the aim of creating social impact. Universities are instances of social change agents [10]. Therefore, most studies have been focused on a situation in which players (social change agents) attempt to find the most influential nodes in order to maximize the total number of infected nodes at the end of the diffusion process $[5,7,8]$. The influence maximization problem has been studied from two perspectives. One perspective occurs in a non-competitive situation where there is only one social change agent who wants to diffuse its own option in a social network. It was first defined by Domingos and Richardson [7], who considered this problem in a 
probabilistic context and provided heuristics to find an influence maximization set. Their research was followed up in several studies such as $[1,3,8,11]$. The other perspective is studying the influence maximization problem in a competitive situation where there are two or more social change agents who compete with each other in order to maximize the diffusion of their options in a network. Recently, the competitive diffusion problem has been investigated for a cascade model in [12-14] and for a threshold model in $[8,15]$.

One of the most efficient tools to study the competitive diffusion problem is game theory, which has lately been developed in some studies such as [16-22]. These studies deal with the diffusion process as a strategic game where social change agents are players and compete to maximize the diffusion of their options (for instance, their information or their goods) in the social network. They seek to find the most influential initial nodes so as to maximize the total number of infected nodes. Most of these research endeavors focus on social networks with homogeneous nodes. Also, these studies assume that if a node receives influence from more than one player at a time, it is considered as a gray node, which will not have any efficacy at the subsequent steps of the diffusion process and can be deleted from the network. Moreover, most of the previous research has not paid attention to the way in which data are diffused. These basic assumptions make the models developed in these studies detached from reality. More recently, study [23] regarded the diffusion process as a game played in a network by external agents. Kermani et al. [23] investigated the effect of individual characteristics and message content on the diffusion process. In particular, they supposed that information diffusion takes place within a communication framework such as a cell phone text messaging service. Furthermore, ref. [23] supposed that nodes are heterogeneous and considered the effect of nodes' identity and message content. These considerations make this study more in line with reality compared to other studies. However, it has to be borne in mind that Kermani et al. [23] supposed that individuals have initial tendency (desire) toward the diffused options which does not change over time, while the tendency of individuals in real-world networks may change due to the influence they receive from their neighbors through time. Another assumption that makes this model out of touch with reality is that the content of messages is constant and that players cannot choose their own message content.

The present study deals with the maximization diffusion problem as a strategic game, where the effect of individuals' characteristics (i.e., nodes in the network), message content and the topology of the network are investigated. That is to say, individuals have an initial tendency toward diffused options by different players. Nodes' tendency can change due to the effects of their friends during the diffusion process. Additionally, players can select the content of their own messages. While the previous studies assumed that players only aim to find the most influential initial nodes, in the model developed here players have the additional goal of finding the best message content. To this end, it is assumed that social change agents attempt to maximize the total sum of the tendencies of individuals toward their own diffused options rather than maximizing the total number of infected nodes. To explain the logic behind this consideration, it is necessary to review the the active node concept as used in the literature. A node is called active if it receives a message from one of the agents (players) in the diffusion process. However, it is clear that some of the received messages do not have any effect on a node and cannot convince the inactive node to choose that agent's option. Therefore, it can be concluded that the total number of infected nodes is not the most suitable criterion for optimizing the diffusion process. Hence, in the current paper, a node is called active if there is a received message that can change its own tendency, and players seek to maximize the sum of individuals' tendencies toward their options, i.e., the level of society's desire to choose the options taken by the players is maximized. All of these considerations bring the proposed model closer to reality compared to previous studies.

The remainder of the paper is organized as follows. Section 2 introduces the game and its components. The competitive influence model is presented in Section 3. In Section 4, the proposed model is implemented on a real data set and provides sensitivity analysis. Finally, Section 5 concludes the paper and offers suggestions for future research. 


\section{The Game}

Without loss of generality, in the proposed model, it is assumed that there are two social change agents (players). All of the results can be extended to the games that involve more than two players. Players are out of the network and have two different options to be diffused in the network. $P=\left\{p_{1}, p_{2}\right\}$ denotes the set of players. The network is represented by means of a weighted directed graph $G(N, E)$ in which $N=\{1,2, \cdots, n\}$ indicates the set of the nodes (i.e., individuals in the network) and $E$ is the set of the edges. This network is a messaging network (Short Message System, Telegram, Instagaram, Whats App, ...) in the sense that arc $(i, j)$ shows that node $i$ can send a message to node $j$ and $w_{i, j} \in[01]$ determines the weight of edge $(i, j)$, which represents the effect of node $i$ on node $j$, and if there is no edge between $i$ and $j$, then $w_{i, j}=0$. For node $i \in G$, two sets $I_{i}=\{j \in N \mid(j, i) \in E\}$ and $O_{i}=\{j \in N \mid(i, j) \in E\}$ are defined based on the graph. The nodes are heterogeneous and have different scores, for instance, the initial tendency of node $i$ toward the players' options, is represented by vector $\alpha_{i}=\left(\alpha_{i 1}, \alpha_{i 2}, \cdots, \alpha_{i m}\right)$, where $m$ is the number of the players (agents) and $-1 \leq \alpha_{i j} \leq 1$ shows the tendency of node $i$ toward the option diffused by the agent (player) $j$ which may be a result of information obtained from friends, advertisement, or other channels. It can change over time because of the influence of messages received from neighbors during the diffusion process. Further, the normalized social skill score of each node $i$ is denoted by $\beta_{i}$, which reflects the sociability score of node $i$ in the network which is evaluated by a social skill questionnaire [23]. Lastly, for each node $i$, two thresholds $0 \leq \theta_{l}^{i} \leq \theta_{h}^{i} \leq 1$ are defined, $0 \leq \delta \leq 1$ is a fixed threshold for the network, and $t \in\{1,2, \cdots\}$ denotes a discrete time step. Agents (players) send messages with different content to the network. The content of player $p_{i}$ 's message is denoted by text $t_{p_{i}}=\left\{t_{p_{i 1}}, t_{p_{i 2}}, \ldots, t_{p_{i m}}\right\}$, where $-1 \leq t_{p_{i 1}} \leq 1$ represents the tendency of message text $t_{p_{i}}$ toward player $p_{1}{ }^{\prime}$ s option. Also, $-1 \leq t_{p_{i 2}} \leq 1$ is the tendency of message text $t_{p_{i}}$ toward player $p_{2}$ 's option. It is thought that text $t_{p_{1}}$ should be $\{1,-1\}$ and text $p_{2}$ should be $\{-1,1\}$. Hence, player $p_{i}$ should diffuse a message whose content thoroughly proves the player's own option and strongly disproves its rival's option. However, it will be shown in Section 4 that this is not always true and sometimes based on social conditions, if players act more moderately, they will be more successful in diffusing their options. Contrary to previous studies, in the proposed model, each player $p_{i}$ not only chooses a set of nodes $I \subseteq N$ (based on its budget) but also selects content text $p_{i}$ for its message at the first step. Thus, the strategy set of player $p_{i}$ gives rise to:

$$
S_{p_{i}}=\left\{\left(I, \text { text }_{p_{i}}\right) \mid I \subseteq N, \text { text }_{p_{i}}=\left(t_{p_{i 1}}, t_{p_{i 2}}\right),-1 \leq t_{p_{i 1}}, t_{p_{i 2}} \leq 1\right\}
$$

Then, diffusion will continue on the basis of an influence model, and at the end of the diffusion process, the total sum of social tendency toward the ith player is this player's payoff, which is denoted by $f_{p_{i}}$ and gives rise to:

$$
f_{p_{i}}\left(s_{p_{i}}, s_{-p_{i}}\right)=\sum_{j=1}^{n} \alpha_{j p_{i}}(t), \quad t \longrightarrow \infty
$$

Each player $p_{i}$ tries to select the best initial set and the best message content in order to maximize $f_{p_{i}}$.

\section{The Influence Model}

In this section, the influence maximization problem sets the rules of the game. Based on [24,25], both node personality and message content affect the diffusion process. Therefore, social skill, initial tendency, and message content are considered in this influence model. Diffusion occurs in discrete steps. At the first step $(t=1)$, each social change agent (player) $p_{i}$ selects a set of nodes $I \subseteq N$ (based on its budget) and content text $p_{i}$ for its message to be sent to $I$. Two different states-active and inactive-are allocated to the nodes at each step in order to explain the state of nodes. In this influence model, a node is called active if its tendency has changed by receiving a message and it is 
inactive if it has not received any message or if the received messages cannot change its initial tendency. At the first step, all of the nodes are inactive. Nodes face three different decision-making situations: first, accepting the incoming message such that the node's tendency changes; second, forwarding the message; and third, selecting the target nodes to forward the message to. It should be noted that, at the first step $(t=1)$, nodes receive a message from social change agents (players), but at subsequent steps $(t \geq 2)$, they receive messages from their neighbors.

At step $t=1$, all of the nodes are inactive and the diffusion process starts. Players select the content of their messages and the initial subset nodes and then send their own messages to them. So, at step $t=1$, one of the following events occurs for each node $i$ :

1. Node $i$ does not receive any message from players and remains inactive.

2. Node $i$ receives (only) a message from the $k$ th social change agent. In this case, the effect of the received message on node $i$ depends on the sender's social skill and the consistency of $i$ 's tendency toward the content of the received message. From a mathematical point of view, the magnitude of this effect is calculated by $\left(\beta_{i} \cdot\left(1-\frac{\| \text { text }_{p_{k}}-\alpha_{i}(1) \|_{2}}{2 \sqrt{2}}\right)\right)$. If this value is lower than node $i^{\prime}$ s low threshold $\theta_{l}^{i}$, then it does not influence node $i$ and cannot change its tendency. That is:

$$
\text { If } \beta_{i} \cdot\left(1-\frac{\| \text { text }_{p_{k}}-\alpha_{i}(1) \|_{2}}{2 \sqrt{2}}\right)<\theta_{l}^{i} \text { then } \alpha_{i}(2)=\alpha_{i}(1),
$$

If this value is higher than node $i$ 's low threshold $\theta_{l}^{i}$, then it can change the tendency of node $i$; that is:

$$
\text { If } \beta_{i} \cdot\left(1-\frac{\| \text { text }_{p_{k}}-\alpha_{i}(1) \|_{2}}{2 \sqrt{2}}\right) \geq \theta_{l}^{i} \text { then } \alpha_{i}(2)=\frac{\alpha_{i}(1)+\text { text }_{p_{k}}}{2},
$$

In this case, node $i$ is called active, and if this value is also higher than node $i^{\prime}$ s high threshold $\theta_{h^{\prime}}^{i}$ then node $i$ decides to forward the received message. In order to model this step, variable $x_{i k}(t)$ needs to be defined as below:

$$
x_{i k}(t)=\left\{\begin{array}{cc}
1, & \text { if node } i \text { decides to send message text } t_{P_{k}} \text { in step } t \\
0, & \text { o.w }
\end{array}\right.
$$

So, the following relationship is concluded:

$$
\text { if } \quad \beta_{i} \cdot\left(1-\frac{\| \text { text }_{p_{k}}-\alpha_{i}(1) \|_{2}}{2 \sqrt{2}}\right) \geq \theta_{h}^{i} \text { then } x_{i k}(2)=1 \quad x_{i k^{\prime}}(2)=0 \text {, }
$$

for $\left(k^{\prime} \in P-k\right)$.

3. Node $i$ receives both messages text $t_{1}$ and text $t_{p_{2}}$ from both players. In this case, node $i$ faces a decision-making situation in which it evaluates the influence of both messages and decides how to act by drawing a comparison between these messages. Realistically, node $i$ selects one of the incoming messages based on the its social skill (node $i$ 's social skill) and the consistency of its tendency toward the content of the received messages. The mathematical representation of this situation is as follows:

$$
\begin{aligned}
& \text { If } \beta_{i} \cdot\left(1-\frac{\| \text { text }_{p_{1}}-\alpha_{i}(1) \|_{2}}{2 \sqrt{2}}\right) \geq \theta_{l}^{i} \text { then } z_{i 1}(2)=1 \text { else } z_{i 1}(2)=0, \\
& \text { if } \beta_{i} \cdot\left(1-\frac{\| \text { text }_{p_{2}}-\alpha_{i}(1) \|_{2}}{2 \sqrt{2}}\right) \geq \theta_{l}^{i} \text { then } z_{i 2}(2)=1 \text { else } z_{i 2}(2)=0, \\
& \alpha_{i}(2)=\frac{\alpha_{i}(1)+z_{i 1}(2) \cdot \text { text }_{p_{1}}+z_{i 2}(2) \cdot \text { text }_{p_{2}}}{1+z_{i 1}(2)+z_{i 2}(2)}
\end{aligned}
$$


Also, node $i$ decides to forward message text $y_{y}$ to some of its neighbors if text $t_{y}$ not only fits best with the node's interest but also the magnitude of its effect is greater than $\theta_{h}^{i}$. That is:

$$
\begin{aligned}
& \text { If } \beta_{i} \cdot\left(1-\frac{\| \text { text }_{y}-\alpha_{i}(1) \|_{2}}{2 \sqrt{2}}\right) \geq \theta_{h}^{i} \text { then } x_{i y}(2)=1, x_{i y^{\prime}}(2)=0 \text {, } \\
& \text { if } \beta_{i} \cdot\left(1-\frac{\| \text { text }_{y}-\alpha_{i}(1) \|_{2}}{2 \sqrt{2}}\right)<\theta_{h}^{i} \text { then } x_{i y}(2)=0, x_{i y^{\prime}}(2)=0 \text {, }
\end{aligned}
$$

where $y=\arg \max _{k \in P}\left\{\beta_{i} \cdot\left(1-\frac{\| \text { text }_{k}-\alpha_{i}(1) \|_{2}}{2 \sqrt{2}}\right)\right\}$ and $y^{\prime}=P-\{y\}$.

At the subsequent steps of the diffusion process, nodes will receive messages from their neighbors who have been active and decided to send a message at the previous step. It is to be noted that if node $i$ accepts one of the incoming messages at step $t$ and decides to forward it, it can only do this at step $t+1$, but not at later steps. Let us suppose that at step $t-1$, node $i$ has accepted message text $t_{P_{k}}$ and decided to forward it at step $t$. However, since the cost of forwarding a message is a consideration, node $i$ cannot forward the message to all of its neighbors. Hence, this node selects some of its neighbors as destination nodes (which are inactive and are also better capable of forwarding the received message compared to other nodes). The choice of destination node $j$ by active node $i$ is related to the consistency of $j$ 's tendency toward the content of the forwarded message, the influence of node $i$ on $j$, and $j$ 's social skill. To model the selection of the destination node using mathematical relationships, it is necessary to define variable $y_{i j k}(t)$ as follows:

$$
y_{i j k}(t)=\left\{\begin{array}{lc}
1, & \text { if node i forwards message text } P_{k} \text { to node } j \text { at step } t \\
0, & \text { o.w }
\end{array}\right.
$$

in which $t \in\{1,2, \cdots\},(i, j) \in E$ and $k \in P$. Therefore, the node selection step is modeled as:

$$
\text { if } \beta_{j} \cdot w_{i j} \cdot\left(1-\frac{\| \text { text }_{p_{k}}-\alpha_{j}(t-1) \|_{2}}{2 \sqrt{2}}\right) \geq \delta \text { then } y_{i j k}(t)=1 \text { else } y_{i j k}(t)=0
$$

The patterns of accepting or rejecting an incoming message at step $(t \geq 2)$ are different from the first step. At subsequent steps of the diffusion process, for each inactive node $i \in N$, one of the following situations occurs.

1. It does not receive any message and remains inactive.

2. It receives (only) one type of message from its neighbors; e.g., message text $t_{p_{k}}$. In this case, according to the magnitude of the impact of the received messages (that depends on the senders' influence on $i$, social skill of $i$, and message content), node $i$ decides how to act. That is:

$$
\text { If } \sum_{j \in I_{i}: y_{j i k}(t)=1} w_{j i} \cdot \beta_{i} \cdot\left(1-\frac{\| \text { text }_{p_{k}}-\alpha_{i}(t-1) \|_{2}}{2 \sqrt{2}}\right) \geq \theta_{l}^{i} \text { then } \alpha_{i}(t)=\frac{\alpha_{i}(t-1)+\text { text }_{p_{k}}}{2},
$$

else, the tendency of node $i$ does not change so that $\alpha_{i}(t)=\alpha_{i}(t-1)$. Also, the forwarding decision step can be represented as below:

$$
\text { if } \sum_{j \in I_{i}: y_{j i k}(t)=1} w_{j i} \cdot \beta_{i} \cdot\left(1-\frac{\| \text { text }_{p_{k}}-\alpha_{i}(t-1) \|_{2}}{2 \sqrt{2}}\right) \geq \theta_{h}^{i} \text { then } x_{i k}(t)=1,
$$

where $\left(k^{\prime}=P_{k}\right)$.

3. It receives both messages text $t_{p_{1}}$ and text $t_{p_{2}}$ from its active neighbors. It will encounter a decision-making situation. The message that fits best with the node's tendency and has been 
forwarded by neighbors which have a considerable influence on $i$ will be accepted and changes $i$ 's tendency. That is:

$$
\begin{aligned}
& \text { If } \sum_{j \in I_{i}: y_{j 1}(t)=1} w_{j i} \cdot \beta_{i} \cdot\left(1-\frac{\| \text { text }_{p_{1}}-\alpha_{i}(t-1) \|_{2}}{2 \sqrt{2}}\right) \geq \theta_{l}^{i} \text { then } z_{i 1}(t)=1 \text { else } z_{i 1}(t)=0, \\
& \text { if } \sum_{j \in I_{i}: y_{j i 2}(t)=1} w_{j i} \cdot \beta_{i} \cdot\left(1-\frac{\| \text { ext }_{p_{2}}-\alpha_{i}(t-1) \|_{2}}{2 \sqrt{2}}\right) \geq \theta_{l}^{i} \text { then } z_{i 2}(t)=1 \text { else } z_{i 2}(t)=0, \\
& \quad \alpha_{i}(t)=\frac{\alpha_{i}(t-1)+z_{i 1}(t) \cdot \text { text }_{p_{1}}+z_{i 2}(t) \cdot \text { text }_{p_{2}}}{1+z_{i 1}(t)+z_{i 2}(t)},
\end{aligned}
$$

Moreover, the forwarding decision step is mathematically shown below:

$$
\begin{aligned}
& \text { If } \sum_{j \in I_{i}: y_{j i q}(t)=1} w_{j i} \cdot \beta_{i} \cdot\left(1-\frac{\| \text { text }_{q}-\alpha_{i}(t-1) \|_{2}}{2 \sqrt{2}}\right) \geq \theta_{h}^{i} \text { then } x_{i q}(t)=1, x_{i q^{\prime}}(t)=0 \\
& \text { if } \sum_{j \in I_{i}: y_{j i q}(t)=1} w_{j i} \cdot \beta_{i} \cdot\left(1-\frac{\| \text { text }_{q}-\alpha_{i}(t-1) \|_{2}}{2 \sqrt{2}}\right)<\theta_{h}^{i} \text { then } x_{i q}(t)=0, x_{i q^{\prime}}(t)=0 \text {, }
\end{aligned}
$$

where $q=\arg \max _{k \in P}\left\{\sum_{j \in I_{i}: y_{j i k}(t)=1} w_{j i} \cdot \beta_{i} \cdot\left(1-\frac{\| \text { text }}{k}-\alpha_{i}(t-1) \|_{2}\right)\right\}$ and $q^{\prime}=P-\{y\}$.

\section{Results}

In this section, the performance of the proposed model is evaluated by implementing the game on two different networks: first, a small dataset with 20 nodes which have been selected randomly and then a real dataset with 163 nodes.

Suppose that there are two players that are out of the network and want to diffuse their own options in a random network. All of the parameters $\alpha_{i}(t), \theta_{l}^{i}, \theta_{h}^{i}$ and $\beta_{i}$ have been randomly selected and $\alpha_{i}(t)$ has been selected such that, $\sum_{i=1}^{20} \alpha_{i 1}(1)=-0.2975$ and $\sum_{i=1}^{20} \alpha_{i 2}(1)=0.2272$, meaning that on average individuals do not have much tendency toward any option diffused by the players. The purpose of the players is to maximize the sum of social tendency toward their own options. They select their initial nodes and message content and then forward the messages to the selected nodes. Without loss of generality, suppose that each player selects only one node (numbers of initial nodes is based on the players' budget.). Thus, the strategy set of players is as follows:

$$
\begin{aligned}
& S_{1}=\left\{\left(i,\left\{1, m_{12}\right\}\right) \mid i \in N,-1 \leq m_{1,2} \leq 1\right\} \\
& S_{2}=\left\{\left(i,\left\{m_{2,1}, 1\right\}\right) \mid i \in N,-1 \leq m_{2,1} \leq 1\right\}
\end{aligned}
$$

As was explained in the previous section, $-1 \leq m_{i j} \leq 1$ represents the tendency of player i's message content toward the option diffused by player $j$. Since $m_{i j}$ is closer to -1 , the content of player $i$ 's message strongly disproves player $j$ 's diffused option. Without loss of generality, to simplify the calculations, $m_{i j}$ is considered to be discrete and belongs to $\{-1,-0.9,-0.8, \ldots, 0, \ldots, 0.9,1\}$. The proposed strategic game is implemented on the network, and the Nash equilibrium will be calculated using the concept of best response functions. It is shown that parameters $\theta_{h}^{i} \theta_{l}^{i}$, and $\delta$ have a significant effect on the players' payoff. Table 1 shows how the number of infected nodes and players' payoff varies when $\theta_{h}^{i}$ and $\theta_{l}^{i}$ vary between 0 and 1 , and $\delta$ varies between 0.1 and 0.9 . Based on parameter definition, increasing the value of the parameters decreases the number of infected nodes 
and the players' payoff. In Table 1, players 'payoff, best initial nodes and the number of infected nodes are represented for different values of parameters.

Table 1. Nash equilibrium for a random network.

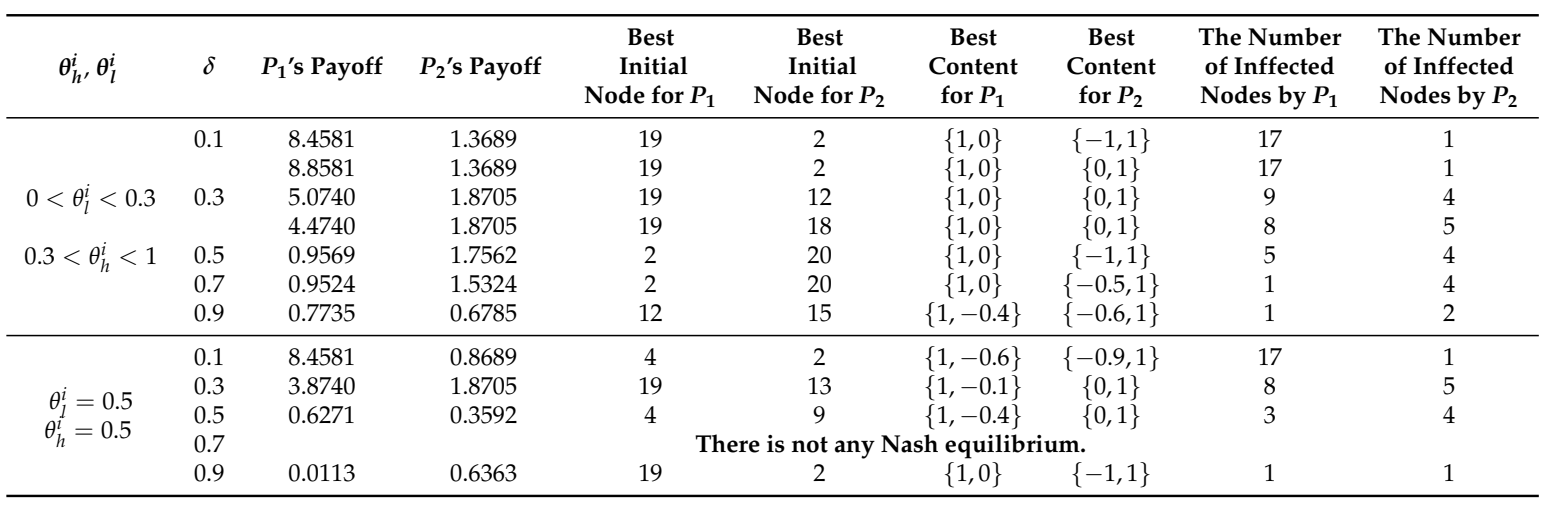

For instance, if $\delta=0.1,0<\theta_{l}^{i}<0.3$ and $0.3<\theta_{h}^{i}<1$, the Nash equilibrium occurs when player 1 selects node 19 and message content $\{1,0\}$. Specifically, the message content of player 1 is such that it strongly promotes the advantage of its option and does not openly attack the credibility of the competitor's option. Player 2 selects node 2 and message content $\{-1,1\}$. Also, player 1's payoff is 8.4581, which is the sum of the tendencies of individuals toward player 1's option at the end of the diffusion process and was -0.2975 prior to the process. Player 2's payoff is 1.3689 , and the number of nodes infected by player 1 and player 2 is 17 and 1, respectively. To show the efficiency of the model in terms of performance validity and solution accuracy, the proposed novel game should be implemented on a real dataset (Abrar data set [6]).

Abrar University is a single-sex university which is located in Tehran, Iran. This data set consists of 163 students enrolled in the fields of computer engineering and industrial engineering in the 2010-2011 and 2011-2012 academic years. These students are regarded as social network nodes $i$ and $j$. Also, a directed link is formed from person $i$ to person $j$ if node $i$ can send a message to node $j$ in a short message system. Sets $I_{i}$ and $O_{i}$ are defined on the basis of the Abrar data set. Further, for each node $i$, the social skill score $\beta_{i}$ is determined based on a questionnaire developed in 1992 [26]. To explain the numerical results, let us assume that two principal cell phone brands (say, Nokia and Samsung) compete with each other to maximize the sales of their products in the network. The purpose of these two agents is to maximize the total tendency of the network (Abrar data set) toward their products and this will maximize the enthusiasm of the nodes to proceed and choose the products of the agents at the time of purchase. The strategy of the players is viral marketing, which is based on a messaging system. This system can be a short messaging system or any online social network such as Telegram, Instagram, WhatsApp, and Facebook. $P=\left\{P_{1}, P_{2}\right\}$ is the set of players. Without loss of generality, let us suppose that each player $p_{k}$ selects one of the students as its initial node because of its budget constraint and also selects content $m_{k}=\left\{m_{k 1}, m_{k 2}\right\}$ for its message to be sent to its chosen initial node $i$. Thus, the strategy set of each player is as follows:

$$
S_{P_{1}}=\left\{\left(i,\left\{1, m_{12}\right\}\right) \mid i \in N,-1 \leq m_{1,2} \leq 1\right\}, S_{P_{2}}=\left\{\left(i,\left\{m_{2,1}, 1\right\}\right) \mid i \in N,-1 \leq m_{2,1} \leq 1\right\}
$$

For each node $i$, initial tendency $\alpha_{i}(1)$, thresholds $\theta_{h}^{i}$, and $\theta_{l}^{i}$ are determined randomly such that $\sum_{i=1}^{163} \alpha_{i 1}=2.0747$ and $\sum_{i=1}^{163} \alpha_{i 2}=15.4826$. Moreover, without loss of generality, $w_{i, j}$ for all $(i, j \in N)$ is supposed to be 1 if there is a link from $i$ to $j$; it is supposed to be 0 if there is no such link.

It is shown that parameters $\theta_{h}^{i}, \theta_{l}^{i}$, and $\delta$ have a significant effect on the players' payoff. The number of infected nodes and also the sum of tendencies of the individuals vary as a function of $\theta_{h}^{i}, \theta_{l}^{i}$ and $\delta$. The Nash equilibrium will be determined for each game. Table 2 shows how the number of infected nodes and players' payoff vary when $\theta_{h}^{i}$ varies between 0.4 and $1, \theta_{l}^{i}$ varies between 0 and 1 , and $\delta$ varies between 0.1 and 0.9 . Based on parameter definition, increasing the value of the parameters 
decreases the number of infected nodes and the players' payoff. Note that, in this game (similar to the previous game) the message content is considered to be discrete and the Nash equilibrium can be calculated using the concept of the best response function. In Table 2, the Nash equilibrium is determined and it is shown that players' payoff varies and the number of infected nodes decreases with an increase in the value of the parameters.

Table 2. Nash equilibrium for different parameters for the Abrar data set.

\begin{tabular}{|c|c|c|c|c|c|c|c|c|c|}
\hline$\theta_{h}^{i}, \theta_{l}^{i}$ & $\delta$ & $P_{1}$ 's Payoff & $P_{2}$ 's Payoff & $\begin{array}{c}\text { Best } \\
\text { Initial } \\
\text { Node for } P_{1}\end{array}$ & $\begin{array}{c}\text { Best } \\
\text { Initial } \\
\text { Node for } P_{2}\end{array}$ & $\begin{array}{c}\text { Best } \\
\text { Content } \\
\text { for } P_{1}\end{array}$ & $\begin{array}{c}\text { Best } \\
\text { Content } \\
\text { for } P_{2}\end{array}$ & $\begin{array}{l}\text { The Number } \\
\text { of Inffected } \\
\text { Nodes by } P_{1}\end{array}$ & $\begin{array}{l}\text { The Number } \\
\text { of Inffected } \\
\text { Nodes by } P_{2}\end{array}$ \\
\hline \multirow{6}{*}{$\begin{array}{c}\theta_{l}^{i}=0 \\
\theta_{h}^{i}=0.4\end{array}$} & 0.1 & 23.3373 & 59.7413 & 81 & 23 & $\{1,0\}$ & $\{-0.6,1\}$ & 87 & 76 \\
\hline & 0.3 & 26.6065 & 54.7455 & 81 & 17 & $\{1,0\}$ & $\{-0.6,1\}$ & 93 & 65 \\
\hline & 0.5 & \multicolumn{8}{|c|}{ There is not any Nash equilibrium. } \\
\hline & 0.7 & 3.5958 & 17.1273 & 27 & 113 & $\{1,-0.5\}$ & $\{-0.2,1\}$ & 12 & 17 \\
\hline & 0.9 & 2.7059 & 16.3655 & 125 & 37 & $\{1,-0.5\}$ & $\{-0.2,1\}$ & 1 & 2 \\
\hline & & 2.7059 & 16.6155 & 125 & 37 & $\{1,0\}$ & $\{-0.2,1\}$ & 1 & 2 \\
\hline \multirow{11}{*}{$\begin{array}{l}0<\theta_{l}^{i}<0.4 \\
0.4<\theta_{h}^{i}<1\end{array}$} & 0.1 & 14.537 & 32.2413 & 93 & 39 & $\{1,-0.5\}$ & $\{-0.6,1\}$ & 77 & 86 \\
\hline & & 31.537 & 32.2413 & 93 & 25 & $\{1,-0.5\}$ & $\{-0.2,1\}$ & 76 & 87 \\
\hline & 0.3 & 21.0968 & 39.0414 & 89 & 23 & $\{1,-1\}$ & $\{-0.2,1\}$ & 60 & 93 \\
\hline & & 21.0968 & 39.0414 & 89 & 28 & $\{1,-1\}$ & $\{-0.2,1\}$ & 60 & 93 \\
\hline & 0.5 & 23.3014 & 33.7797 & 22 & 3 & $\{1,0\}$ & $\{-0.2,1\}$ & 68 & 59 \\
\hline & & 23.8083 & 33.7797 & 22 & 49 & $\{1,0\}$ & $\{-0.2,1\}$ & 68 & 59 \\
\hline & 0.7 & 3.2396 & 16.7611 & 35 & 93 & $\{1,-0.5\}$ & $\{-0.2,1\}$ & 12 & 13 \\
\hline & & 3.8620 & 11.1159 & 84 & 46 & $\{1,-0.5\}$ & $\{-0.2,1\}$ & 12 & 11 \\
\hline & & 3.2396 & 17.1159 & 84 & 93 & $\{1,-0.5\}$ & $\{-0.2,1\}$ & 10 & 13 \\
\hline & 0.9 & 3.1313 & 16.7649 & 54 & 144 & $\{1,-0.5\}$ & $\{-0.6,1\}$ & 2 & 2 \\
\hline & & 3.5313 & 16.7649 & 54 & 144 & $\{1,-0.5\}$ & $\{-0.2,1\}$ & 2 & 2 \\
\hline \multirow{6}{*}{$\begin{array}{c}\theta_{l}^{i}=0.5 \\
\theta_{h}^{i}=1\end{array}$} & 0.1 & 2.7095 & 16.1442 & 1 & 115 & $\{1,0\}$ & $\{-0.2,1\}$ & 1 & 1 \\
\hline & & 2.7541 & 16.1442 & 1 & 160 & $\{1,-1\}$ & $\{-0.6,1\}$ & 1 & 1 \\
\hline & 0.3 & 2.5541 & 16.1442 & 1 & 160 & $\{1,0\}$ & $\{-1,1\}$ & 1 & 1 \\
\hline & 0.5 & 2.5541 & 16.1442 & 1 & 160 & $\{1,0\}$ & $\{-1,1\}$ & 1 & 1 \\
\hline & 0.7 & 2.5541 & 16.1442 & 1 & 160 & $\{1,0\}$ & $\{-1,1\}$ & 1 & 1 \\
\hline & 0.9 & 2.5541 & 15.8623 & 10 & 160 & $\{1,0\}$ & $\{-1,1\}$ & 1 & 1 \\
\hline$\theta_{l}^{i}=1, \theta_{h}^{i}=1$ & \multicolumn{9}{|c|}{ The diffusion process does not happen. } \\
\hline
\end{tabular}

As is shown in Table 2, some of the games have no Nash equilibrium, some have only one, and some others have more than one. For instance, if $\delta=0.7, \theta_{l}^{i}=0$ and $\theta_{h}^{i}=0.4$, the Nash equilibrium occurs when player 1 selects node 27 and message content $\{1,-0.5\}$. Specifically, the message content of player 1 is such that it strongly promotes the advantage of its option and does not openly attack the credibility of the competitor's option, but rather mildly expresses the disadvantages of the competitor's option. Player 2 selects node 113 and message content $\{-0.2,1\}$. Also, player 1's payoff is 3.5958, which is the total sum of the tendencies of individuals toward player 1's option at the end of the diffusion process and was 2.0747 prior to the process. Player 2's payoff is 17.1273 , and the number of nodes infected by player 1 and player 2 is 12 and 17, respectively. By definition, the Nash equilibrium occurs in strategy profiles where players have no motivation to perform differently from the Nash equilibrium. In the present study, the players' strategy is to find the best initial nodes and the best message content. Below comes an analysis of a situation where at least one of the players deviates from the Nash equilibrium, once for selecting the initial node and the other time for selecting message content. For example, when $\delta=0.7,0 \leq \theta_{l}^{i} \leq 0.4$, and $0.4 \leq \theta_{h}^{i} \leq 1$, the performance of the competitive influence model propounded here is compared with some of the well-known models previously proposed in the literature (e.g., MGBD, MGEB, MGTB, MGSB, and MRND [23]), and the numerical results are summarized in Tables 4 and 5 . Table 3 shows the initial node determined by the above-mentioned models.

Table 3. Suggested initial nodes based on different strategies.

\begin{tabular}{lccccc}
\hline & MGDB & MGTB & MGSB & MGEB & MRND \\
\hline Player 1 & 30 & 30 & 157 & 30 & 25 \\
Player 2 & 85 & 85 & 32 & 32 & 88 \\
\hline
\end{tabular}


In Table 4, it is assumed that player 1 deviates from the Nash equilibrium and selects the initial node based on the above-cited strategies. It follows that its payoff will decrease in comparison with the Nash equilibriums obtained in Table 2. Therefore, it does not have any motivation for deviation.

Table 4. Nash equilibrium when player 1 performs based on some well-known strategies.

\begin{tabular}{|c|c|c|c|c|c|c|c|c|}
\hline \multicolumn{2}{|c|}{$\begin{array}{l}\text { Initial Node for } \\
P_{1} \text { Based on } \\
\text { Well-Known } \\
\text { Strategies }\end{array}$} & $P_{1}$ 's Payoff & $P_{2}$ 's Payoff & $\begin{array}{l}\text { Best } \\
\text { initial } \\
\text { Node } \\
\text { for } P_{2} \\
\end{array}$ & $\begin{array}{c}\text { Best } \\
\text { Content } \\
\text { for } P_{1}\end{array}$ & $\begin{array}{c}\text { Best } \\
\text { Content } \\
\text { for } P_{2}\end{array}$ & $\begin{array}{c}\text { The Number } \\
\text { of Inffected } \\
\text { Nodes by } \\
P_{1}\end{array}$ & \multirow{2}{*}{$\begin{array}{c}\text { The Number } \\
\text { of Inffected } \\
\text { Nodes by } \\
P_{2}\end{array}$} \\
\hline MGDB & & 1.8135 & 16.4623 & 46 & $\{1,-1\}$ & $\{-0.2,1\}$ & 0 & \\
\hline MGTB & 30 & 1.8135 & 16.4623 & 46 & $\{1,-0.5\}$ & $\{-0.2,1\}$ & 0 & 2 \\
\hline MGEB & & 1.8135 & 16.4623 & 46 & $\{1,0\}$ & $\{-0.2,1\}$ & 0 & 2 \\
\hline \multirow{3}{*}{ MGSB } & \multirow{3}{*}{157} & 2.3033 & 16.2556 & 46 & $\{1,-1\}$ & $\{-0.2,1\}$ & 1 & 2 \\
\hline & & 2.3033 & 16.5056 & 46 & $\{1,-0.5\}$ & $\{-0.2,1\}$ & 1 & 2 \\
\hline & & 2.3033 & 16.7556 & 46 & $\{1,0\}$ & $\{-0.2,1\}$ & 1 & 2 \\
\hline \multirow{3}{*}{ MRND } & \multirow{3}{*}{25} & 1.8164 & 16.1319 & 46 & $\{1,-1\}$ & $\{-0.2,1\}$ & 1 & 2 \\
\hline & & 1.8164 & 16.1319 & 46 & $\{1,-0.5\}$ & $\{-0.2,1\}$ & 1 & 2 \\
\hline & & 1.8164 & 16.1319 & 46 & $\{1,0\}$ & $\{-0.2,1\}$ & 1 & 2 \\
\hline
\end{tabular}

Similarly, in Table 5, it is assumed that player 2 deviates from the Nash equilibrium and performs on the basis of the strategies cited in Table 3. In this case, the payoff of this palyer will decrease, and so it will not be motivated to deviate.

Table 5. Nash equilibrium when player 2 performs based on some well-known strategies.

\begin{tabular}{|c|c|c|c|c|c|c|c|}
\hline $\begin{array}{c}\text { Initial Node for } \\
P_{2} \text { Based on } \\
\text { Well-Known } \\
\text { Strategies }\end{array}$ & $P_{1}^{\prime}$ 's Payoff & $P_{2}$ 's Payoff & $\begin{array}{l}\text { Best } \\
\text { Initial } \\
\text { Node } \\
\text { for } P_{1}\end{array}$ & $\begin{array}{c}\text { Best } \\
\text { Content } \\
\text { for } P_{1}\end{array}$ & $\begin{array}{c}\text { Best } \\
\text { Content } \\
\text { for } P_{2}\end{array}$ & $\begin{array}{c}\text { The Number } \\
\text { of Inffected } \\
\text { Nodes by } \\
P_{1}\end{array}$ & $\begin{array}{c}\text { The number } \\
\text { of Inffected } \\
\text { Nodes by } \\
P_{2}\end{array}$ \\
\hline $\begin{array}{l}\text { MGDB } \\
\text { MGTB }\end{array}$ & $\begin{array}{l}4.0984 \\
4.2984 \\
4.4684\end{array}$ & $\begin{array}{l}15.1517 \\
15.1517 \\
15.1517\end{array}$ & $\begin{array}{l}35 \\
35 \\
35\end{array}$ & $\begin{array}{l}\{1,-0.5\} \\
\{1,-0.5\} \\
\{1,-0.5\}\end{array}$ & $\begin{array}{c}\{-1,1\} \\
\{-0.6,1\} \\
\{-0.2,1\}\end{array}$ & $\begin{array}{l}14 \\
14 \\
14\end{array}$ & $\begin{array}{l}1 \\
1 \\
1\end{array}$ \\
\hline $\begin{array}{l}\text { MGSB } \\
\text { MGEB }\end{array}$ & 4.1371 & 15.3215 & 35 & $\{1,-0.5\}$ & $\{-0.2,1\}$ & 14 & 8 \\
\hline MRND & 3.7802 & 14.4450 & 46 & $\{1,-0.5\}$ & $\{-0.2,1\}$ & 14 & 2 \\
\hline
\end{tabular}

The solutions obtained in Tables 4 and 5 follow the definition of the Nash equilibrium in the sense that if one of the players deviates from the Nash equilibrium and chooses one of the aforementioned well-known strategies when selecting initial nodes, its payoff will decrease, and so it is not motivated to deviate. Kermani et al. [23] considered message content to be fixed in their influence model (i.e., $\{1,-1\}$ for player 1 and $\{-1,1\}$ for player 2$)$. However, the present study supposed for the first time that players select the content of their messages as well as selecting the initial nodes. As Table 2 shows, it is worth noting that in some instances the Nash equilibrium occurs in strategy profiles where the content of the messages is not necessarily $\{1,-1\}$ or $\{-1,1\}$. In order to show the superiority of the proposed model to the model developed by Kermani et al. [23], when $\delta=0.5, \delta=0.7,0 \leq \theta_{l}^{i}<0.4$ and $0.4 \leq \theta_{h}^{i}<1$, it is supposed that at least one of the players performs according to Kermani's model and selects fixed content for its own message $(\{1,-1\}$ for player 1 and $\{-1,1\}$ for player 2$)$. Under this assumption, the Nash equilibrium is achieved for the proposed game in Table 6, and it is concluded that if the content of the messages for at least one of the players becomes fixed based on [23], that player's payoff and also the number of infected nodes will decrease.

Based on the results in Tables 2 and 6 , if $\delta=0.5$ and the message content of player 1 becomes constant to $\{1,-1\}$, its payoff decreases from 23.3014 to 4.5542 and also the total number of infected nodes will decreases from 127 to 115 . Therefore, if players can select their message content in addition to selecting an initial set of nodes, they can be successful at the diffusion process. 
Table 6. Nash equilibrium when at least one of the players selects fixed content.

\begin{tabular}{ccccccccc}
\hline & & & & $\begin{array}{c}\text { Best } \\
\text { Initial } \\
\text { Node } \\
\text { for } \boldsymbol{P}_{\mathbf{1}}\end{array}$ & $\begin{array}{c}\text { Best } \\
\text { Initial } \\
\text { Node } \\
\text { for } \boldsymbol{P}_{\mathbf{2}}\end{array}$ & $\begin{array}{c}\text { Best } \\
\text { Content } \\
\text { for } \\
\text { Comprtitor }\end{array}$ & $\begin{array}{c}\text { The Number } \\
\text { of Inffected } \\
\text { Nodes by } \\
\boldsymbol{P}_{\mathbf{1}}\end{array}$ & $\begin{array}{c}\text { The Number } \\
\text { of Inffected } \\
\text { Nodes by } \\
\boldsymbol{P}_{\mathbf{2}}\end{array}$ \\
\hline $\begin{array}{c}\text { Player 1 } \\
\text { selects }\end{array}$ & 0.5 & 4.5542 & 28.3361 & 22 & 48 & $\{-0.2,1\}$ & 31 & 82 \\
$\{1,-1\}$ & 0.7 & 2.5542 & 29.3747 & 41 & 48 & $\{-0.2,1\}$ & 31 & 84 \\
\hline & & -1.9469 & 19.4809 & 135 & 84 & $\{1,-1\}$ & 36 & 47 \\
Player 2 & 0.5 & -1.9469 & 19.1998 & 158 & 84 & $\{1,-1\}$ & 36 & 47 \\
selects & & -1.9469 & 19.1994 & 158 & 84 & $\{1,0\}$ & 36 & 47 \\
$\{-1,1\}$ & 0.7 & 3.7878 & 16.2260 & 84 & 66 & $\{1,0\}$ & 12 & 2 \\
& & 3.2051 & 16.2260 & 84 & 144 & $\{1,0\}$ & 12 & 4 \\
\hline
\end{tabular}

In Tables 4-6, some useful comparisons have been made to show the efficiency of the proposed model. According to the definitions, the Price Of Anarchy (POA) is a concept in economics and game theory that measures how the efficiency of a system degrades due to selfish behavior of its agents. It is a general notion that can be extended to diverse systems and notions of efficiency. So, in the following, to better consider the efficiency of the outcomes, the price of anarchy for the solutions will be calculated first, and then account will be taken of two settings in which players send their message to the network separately as in a one-player game. To calculate the price of anarchy, we define a measure of efficiency of each outcome that is called welfare function; Welf : $\mathbf{S} \longrightarrow \mathbf{R}$ where $S$ is the set of strategy profiles and $\operatorname{Welf}(\mathbf{s})=\sum_{\mathbf{i} \in \mathbf{N}} \mathbf{u}_{\mathbf{i}}(\mathbf{s})$. Suppose that NE denotes the set of Nash equilibrium of the game. The Price of Anarchy is then defined as:

$$
P O A=\frac{\max _{s \in S} \operatorname{Welf}(s)}{\min _{s \in N E} \operatorname{Welf}(s)}
$$

The price of anarchy is shown in Table 7 for the proposed game in case $\theta_{l}^{i}=0$ and $\theta_{h}^{i}=0.4$; $\delta$ varies between 0.1 and 0.9 .

Table 7. Price Of Anarchy.

\begin{tabular}{cccccc}
\hline$\delta$ & POA & $\boldsymbol{P}_{\mathbf{1}}$ 's Payoff & $\boldsymbol{P}_{\mathbf{2}}{ }^{\prime}$ s Payoff & $\boldsymbol{s}_{\mathbf{1}}$ & $\boldsymbol{s}_{\mathbf{2}}$ \\
\hline 0.1 & 1.087 & 82.5373 & 7.7413 & $(1,\{1,0\})$ & $(1,\{-1,1\})$ \\
0.3 & 1.022 & 67.7967 & 15.3790 & $(54,\{1,0\})$ & $(101,\{-0.2,1\})$ \\
0.5 & NON & 29.1317 & 25.2867 & $(54,\{1,0\})$ & $(76,\{-0.2,1\})$ \\
0.7 & 1.118 & 3.99 & 19.1732 & $(76,\{1,0\})$ & $(54,\{-0.2,01\})$ \\
0.9 & 1.059 & 3.4349 & 16.7610 & $(76,\{1,0\})$ & $(54,\{-0.2,01\})$ \\
\hline
\end{tabular}

Based on the results in Table 7, when delta $=0.1$, Nash equilibrium is achieved in Table 2 such that the payoff of players 1 and 2 is 23.3373 and 59.7413 respectively and is achieved when player 1 selects $(81,\{1,0\})$ and player 2 selects $(23,\{-0.6,1\})$. In this case, the sum of utilities is $23.3373+59.7413=83.0786$, but if players prefer the collective utility to individual utility then they act differently from Nash equilibrium; based on Table 7 , player 1 selects $(1,\{1,0\})$ and player 2 selects $(1,\{-1,1\})$ and the sum of utilities increases to $1.087+82.5373=90.2787$. The ratio of these amounts of utilities shows the price of anarchy for the game. Note that when $\delta=0.5$, the Nash equilibrium does not exist, but if players try to maximize the overall utility, they select $(54,\{1,0\})$ and $(76,\{-0.2,1\})$ and this utility will be 54.4183 , thus in this case, the price of anarchy has not been calculated. Based on the results in Table 7, the price of anarchy is larger than 1 (although this difference is not very great), and does not relate to parameter $\delta$, since $\delta$ increases; POA increases in some parts and decreases in others. 
Now, suppose that players send their message separately to the network and they play in a noncompetitive situation in the absence of rivals. Certainly, their payoff is expected to increase compared to competitive conditions. This consideration is implemented on the proposed game and the results are shown in Tables 8 and 9, respectively.

Table 8. Only player 1 forwarding its message to the network.

\begin{tabular}{ccccc}
\hline$\delta$ & Payoff & Best Initial Node & Best Message Content & The Number of Infected Nodes \\
\hline 0.1 & 82.5373 & $1,2,10$ & $\{1,-0.5\}$ & 163 \\
0.3 & 64.9419 & 125 & $\{1,0\}$ & 144 \\
0.5 & 24.2832 & 76 & $\{1,0\}$ & 84 \\
0.7 & 4.6257 & 31 & $\{1,-0.5\}$ & 14 \\
0.9 & 3.0649 & 125 & $\{1,-0.5\}$ & 1 \\
\hline
\end{tabular}

Table 9. Only player 2 forwarding its message to the network.

\begin{tabular}{ccccc}
\hline$\delta$ & Its Payoff & Best Initial Node & Best Message Content & The Number of Infected Nodes \\
\hline 0.1 & 89.2413 & $92,1,17$ & $\{-0.6,1\}$ & 163 \\
0.3 & 72.7808 & 75 & $\{-0.2,1\}$ & 145 \\
0.5 & 36.1180 & 75 & $\{-0.2,1\}$ & 85 \\
0.7 & 18.0687 & 107 & $\{-0.2,1\}$ & 15 \\
0.9 & 16.4780 & 37 & $\{-0.2,1\}$ & 2 \\
\hline
\end{tabular}

As is obvious in Tables 8 and 9, if players send their message to the network in the absence of competitors, they can diffuse their option better than in a competitive situation. For instance, if $\delta=0.1$ then player 1's payoff is 23.3373 and player 2's payoff is 59.7413 in the competitive game (Table 2). However, based on Tables 8 and 9, when they play the game separately, their payoff is 82.5373 and 89.2413 , respectively. These results are exactly what we expect to achieve. This is another increase that shows the efficiency and good performance of the proposed model.

\section{Conclusions}

In this paper, we developed a novel game theory model to study the influence maximization problem in a messaging network. The proposed model is superior to the ones previously reported owing to the assumptions made in the present study for the network and also because of the realistic nature of the diffusion method employed. More particularly, unlike in the previous studies, we assumed that individuals in the network are heterogeneous (i.e., that they have personal tendencies that can change over time) and have different degrees of influence on their neighbors. The proposed model also takes account of information content. The players attempt to find the best initial set of nodes and the best content in order to maximize the total sum of network tendencies toward their options, whereas in the previous studies the sole purpose of the players was to maximize the total number of infected nodes. A possible avenue of research in the future is to model this influence maximization problem as a multi-objective linear optimization problem and solve it through exact methods. Another interesting possibility would be to explore networks other than messaging networks, finding mixed-strategy Nash equilibrium instead of the pure-strategy Nash equilibrium.

Author Contributions: All authors contributed equally to this manuscript.

Conflicts of Interest: The authors declare no conflict of interest. 


\section{References}

1. Easley, D.; Kleinberg, J. Networks, Crowds, and Markets; Cambrige University Press: Cambrige, UK, 2010.

2. Goyal, S. Connections: An Introduction to the Economics of Networks; Princeton University Press: Princeton, NJ, USA, 2007.

3. Jackson, M. Social and Economic Networks; Princeton University Press: Princeton, NJ, USA, 2008.

4. Mahini, M.; Dehghan, M.; Navidi, H.; Masoud Rahmani, A. GaMe-PLive: A new game theoric mechanism for P2P live video streaming. Int. J. Commun. Syst. 2016, 29, 1187-1203.

5. Kheirkhah, M.; Navidi, H.; Messi Bidgoli, M. A bi-level network interdiction model for solving the hazmat routing problem. Int. J. Prod. Res. 2016, 54, 459-471.

6. Kermani, M.; Karimimajd, A.; Mohammadi, N.; Aliahmadi, B. A note on predicting how people intract in attributed social networks. Int. J. Curr. Life Sci. 2014, 4, 2510-2514.

7. Domingos, P.; Richardson, M. Mining the network value of customers. In Proceedings of the 7th ACM SIGKDD International Conference of KDD, San Francisco, CA, USA, 26-29 August 2001; pp. 57-66.

8. Kempe, D.; Kleinberg, J.; Tardos, E. Maximizing the spread of influence in a social nerwork. In Proceedings of the 9th ACM SIGKDD International Conference on KKD, Washington, DC, USA, 24-27 August 2003; pp. 137-146.

9. De Bruyn, A.; Lilien, G.L. A multi-stage model of word-of-mouth influence through viral marketing. Int. J. Res. Mark. 2008, 25, 151-163.

10. Weinberg, A.S. The University: An agent of social change? Qual. Soc. 2002, 25, 263-272.

11. Chen, N. On the approximability of influence in social networks. SIAM J. Discrete Math. 2009, 23, $1400-1415$.

12. Bharathi, S.; Kempe, D.; Salek, M. Competitive influence maximization in social networks. In Proceedings of the Internet and Network Economics (IWINE), San Diego, CA, USA, 12-14 December 2007; pp. 306-311.

13. Carnes, T.; Nagaragan, C.; Wild, S.M.; Van Zuylen, A. Maximizing influence in a competitive social network, A follower's perspective. In Proceedings of the 9th International Conference on Electronic Commerce (ICEC), Minneapolis, MN, USA, 19-22 August 2007; pp. 351-360.

14. Kostka, J.; Wattenhofer, R. Word of mouth: Rumor dissemination in social networks. In Proceedings of the 15th International Colloquium on SIROCCO, Villars-sur-Ollon, Switzerland, 17-20 June 2008; pp. 185-196.

15. Borodin, A.; Filmus, Y.; Oren, J. Threshold models for competitive influence in social networks. In Proceedings of the Internet and Network Economics (IWINE), Stanford, CA, USA, 13-17 December 2010; pp. 539-550.

16. Immor, N.; Kleinberg, J.M.; Mahdian, M.; Wexler, T. The role of compatibility in the diffusion of technologies through social networks. In Proceedings of the 8th ACM Confrence on Electronic Commerce, San Diego, CA, USA, 11-15 June 2007; pp. 75-83.

17. Tzoumas, V.; Amantidis, C.; Markakis, E. A game theoric analysis of a competitive diffusion process over social networks. In Proceedings of the 8th Internet and Network Economics (WINE), Liverpool, UK, 9-12 December 2012; Volume 7695, pp. 1-14.

18. Agha Mohamad Ali Kermani, M.; Aliahmadi, A.; Honeman, R. Optimizing the choice of influential nodes for diffusion on a social network. Int. J. Commun. Syst. 2016, 29, 1235-1250.

19. Alon, N.; Feldman, M.; Procaccia, A.D.; Tennenholtz, M. A note on competitive diffusion through social networks. Inf. Proc. Lett. 2010, 110, 221-225.

20. Goyal, S.; Kearns, M. Competitive contagion in networks. In Proceedings of the 44th Annual ACM Symposium on Theory of Computing (STOC), New York, NY, USA, 19-22 May 2012; pp. 759-774.

21. Morris, S. Contagion. Rev. Econ. Stud. 2000, 67, 57-78.

22. Mossel, E.; Roch, S. On the submodularity of influence in social networks. In Proceedings of the Thirty-Ninth Annual ACM Symposium on Theory of Computing (STOC), San Diego, CA, USA, 11-13 June 2007; pp. 128-134.

23. Agha Mohamad Ali Kermani, M.; Ardestani, S.F.F.; Aliahmadi, A.; Barzinpour, R.F. A novel game theoric approach for modeling competitive information diffusion in social networks heterogeneous nodes. Phys. A Stat. Mech. Appl. 2017, 466, 570-582.

24. Son, I.; Lee, D.; Kim, Y. Understanding the effect of message content and user identity on information diffusion on online social networks. In Proceedings of the Pacific Asia Conference on Information Systems (PACIS), Jeju Island, Korea, 18-22 June 2013; p. 8. 
25. Weng, L. Information Diffusion on Online Social Networks; Indiana University: Bloomington, IN, USA, 2014.

26. Inderbitzen, H.M.; Foster, S.L. The teenage inventory of social skills: Development, reliability, and validity. Psychol. Assess. 1992, 4, 451.

(C) 2018 by the authors. Licensee MDPI, Basel, Switzerland. This article is an open access article distributed under the terms and conditions of the Creative Commons Attribution (CC BY) license (http://creativecommons.org/licenses/by/4.0/). 